\title{
Estrategia integral desde la Universidad Estatal a Distancia para abordar la problemática de la trata de personas
}

\section{Nora González Chacón ${ }^{1}$ y Gabriela Villalobos Torres ${ }^{2}$}

1. Coordinadora del Programa Agenda Joven, Universidad Estatal a Distancia, San José, Costa Rica.

Correo electrónico: ngonzalez@uned.ac.cr

2. Encargada de la Cátedra de Trabajo Social, Escuela de Ciencias Sociales y Humanidades, Universidad Estatal a Distancia, San José, Costa Rica. Correo electrónico: gvillalobost@uned.ac.cr

\author{
Recibido: Julio 2013 • Aceptado: Mayo 2014
}

\section{RESUMEN}

Para la erradicación de la trata de personas como forma moderna de esclavitud se requiere acciones de diferentes entes, tales como los gobiernos, las organizaciones civiles y de los centros educativos, entre otros muchos actores. La propuesta de este artículo se enfoca en la prevención, la cual se canaliza por medio de la educación a las personas jóvenes, con apoyo de la comunidad educativa y de su entorno social inmediato. Es importante que las personas jóvenes se vean a sí mismas como sujetos integrales de derechos, lo cual implica su ser físico, psicológico y social y se extiende a las áreas que le permitan planificar o crear las condiciones para proyectar y tener una vida plena.

Palabras clave: trata de personas, esclavitud, derechos, autodeterminación, integralidad.

\begin{abstract}
For human trafficking eradication as a modern form of slavery different actions are required from entities such as governments, civil organizations, and schools, among many other actors. The purpose of this article focuses on prevention, which is channeled through education to young people, with support from the educational community, and their immediate social environment. It is important that youngsters see themselves as integral people with rights; this involves their physical, psychological, and social being, and extends to areas that will help them plan or create the conditions to design and have a full life.
\end{abstract}

Key words: human trafficking, slavery, rights, self-determination, integrity.

\section{Introducción}

La trata de personas es una forma moderna de esclavitud. Se alimenta de redes de tráfico cada vez más organizadas, que actúan en cualquier lugar del mundo. Estas redes fomentan el reclutamiento, el tráfico y el comercio de las personas para distintos fines, según sea el mercado de oferta y demanda.
El artículo 3, inciso a) del Protocolo de Palermo (2003) define "trata de personas" de la siguiente manera:

Por trata de personas se entenderá la captación, el transporte, el traslado, la acogida o la recepción de personas, recurriendo a la amenaza o al uso de la fuerza u otras formas de coacción, al rapto, al fraude, al engaño, al abuso de poder o de una situación 
de vulnerabilidad o a la concesión o recepción de pagos o beneficios para obtener el consentimiento de una persona que tenga autoridad sobre otra, con fines de explotación. Esa explotación incluirá, como mínimo, la explotación de la prostitución ajena $u$ otras formas de explotación sexual, los trabajos o servicios forzados, la esclavitud o las prácticas análogas a la esclavitud, la servidumbre o la extracción de órganos.

La trata de personas se refiere a toda persona de cualquier sexo, edad y procedencia que realiza individual o grupalmente-o como parte de una red nacional o internacional y con pleno conocimiento de las consecuencias de sus actos- las siguientes conductas típicas: captación o reclutamiento de personas para ser tratadas y el traslado, el transporte, la retención o la recepción de víctimas.

Hay varios instrumentos de derecho internacional que sancionan este delito, siendo los siguientes trece los más representativos: Convención relativa a la esclavitud; Protocolo que enmienda la Convención sobre la esclavitud; Convención suplementaria sobre la abolición de la esclavitud, la trata de esclavos y las instituciones y prácticas análogas a la esclavitud; Convención de las Naciones Unidas contra la delincuencia organizada transnacional (Convención de Palermo); Protocolo contra el tráfico ilícito de migrantes por tierra, mar y aire, que complementa la Convención de las Naciones Unidas contra la delincuencia organizada transnacional (Protocolos de Palermo); Protocolo para prevenir, reprimir y sancionar la trata de personas, especialmente mujeres y niños, que complementa los Protocolos de Palermo; Convenio para la represión de la trata de personas y de la explotación de la prostitución ajena y protocolo final; Convención internacional para la represión de la trata de mujeres y menores; Convención internacional relativa a la represión de la trata de mujeres mayores de edad; Protocolo que enmienda la Convención para la supresión del tráfico de mujeres y niños, concluida en Ginebra el 30 de septiembre de 1921; Convención para la supresión del tráfico de mujeres mayores de edad, concluida en Ginebra el 11 de octubre de 1933; Convenio internacional para la supresión del tráfico de trata de blancas, firmado en París el 18 de mayo de 1910, enmendado por el protocolo firmado en Lake Success, Nueva York, el 4 de mayo de 1949; Acuerdo internacional para la supresión del tráfico de trata de blancas, firmado en París el 18 de Mayo de 1904, enmendado por el protocolo firmado en Lake Success, Nueva York, el 4 de Mayo de 1949; y Plan de acción mundial de las Naciones Unidas para combatir la trata de personas.

Los fines de la trata son muy diversos e incluyen la explotación sexual y laboral, la adopción ilegal, la mendicidad, el tráfico de órganos, la guerra, el trabajo forzoso, la servidumbre por deuda, el matrimonio forzado, la prostitución forzosa y en general la esclavitud (Protocolo de Palermo, 2003).

\section{La mercancía: las personas}

La demanda de personas para distintos fines es la raíz del problema de la trata de personas en todas sus manifestaciones. Se demanda una mercancía: el ser humano. El problema se transforma en negocio económico, mercantilista, con un mercado lucrativo en el que redes nacionales o internacionales (con capital corporativo) obtienen ganancias multimillonarias. Se estima que

la trata de personas es un negocio mundial de unos
32.000 millones de dólares al año, siendo el crimen
más lucrativo después del tráfico de drogas, según
estimaciones de la Organización para la Coopera-
ción Económica y el Desarrollo (conocida como la
OECD por sus siglas en inglés). Esto significa que
anualmente entre un millón a dos millones de muje-
res, hombres, niñas y niños son traficadas para fines
de explotación, y los traficantes ganan entre 4.000
y 50.000 dólares por persona, dependiendo del lugar
de origen y destino de la víctima (BID, 2006).

Algunos datos relevantes para dimensionar el tema de la trata de personas en su perspectiva internacional son los siguientes: en Estados Unidos anualmente ingresan entre 14550 y 17500 personas víctimas de trata, distribuidas de la siguiente manera: del sureste asiático cerca de 7000 víctimas, de Latinoamérica 5500 y específicamente 
para la trata con fines de explotación sexual, según el Departamento de Estado de los Estados Unidos, hay 50000 mujeres, niñas y niños explotados sexualmente en el país. Según la Interpol, cada año 35000 mujeres colombianas son víctimas de trata; entre 50000 y 70000 mujeres originarias de República Dominicana trabajan fuera de su país en prostitución; en el 2002 más de 2000 menores centroamericanos mayormente migrantes, fueron encontrados prostituidos en Guatemala. En Brasil, cerca de 500000 niñas y muchas de ellas víctimas de trata se dedican al servicio sexual (prostitución) y al trabajo en las minas de oro de Amazonia. Cada año entre 1000 y 1500 bebés y menores guatemaltecos son víctimas de trata para falsas adopciones en Europa y Estados Unidos. En San José, Costa Rica hay unas 2000 niñas que trabajan en la prostitución, muchas de ellas migrantes. Adolescentes colombianas, dominicanas y de Filipinas han sido llevadas a Costa Rica y a otros destinos conocidos como de turismo sexual como víctimas de trata con fines sexuales (Batista, s.f.: 4).

Todas estas formas de trata implican que a víctimas les sean violentados muchos de sus derechos fundamentales: el derecho a la vida, a la libertad, a no ser esclavizadas, torturadas o sometidas a servidumbre, a trabajos forzosos, a la mendicidad; además se les violenta el pleno ejercicio del derecho a la nacionalidad, a la identidad, a a tener un nombre y a la autodeterminación. Al negárseles su dignidad humana, se les niega su condición de personas, y por ende, todos sus derechos como seres humanos.

Desde la captación hasta la explotación, y la posterior salida de la persona tratada (cuando logra sobrevivir) hay una afectación a la dignidad humana. La víctima es deshumanizada y convertida en un objeto intercambiable, como mercancía y con precio. Su condición de esclava conlleva la pérdida de la capacidad para decidir libremente y de discernir entre lo correcto y lo incorrecto y decidir sobre sus actos y consecuencias. "La autonomía, es, pues, el fundamento de la dignidad de la naturaleza humana y de toda naturaleza racional" (Kant, 1996: 49).

\section{La prevención}

Se trata de impedir que en el futuro ocurra lo mismo, es decir, que se repitan las condiciones que promueven la trata. Al ser este flagelo un problema muy complejo, su intervención depende mucho del lugar desde el cual se desarrolla la estrategia: por un lado, la sociedad civil debe exigir el cumplimiento de sus derechos humanos al Estado y, por otro, los estados deben desarrollar estrategias desde la institucionalidad, que incluyan al Ministerio Público, a los centros educativos, a la comunidad internacional y la intervención educativa hacia las personas para que no demanden de la esclavitud.

Hay nudos problemáticos que son una constante en los diferentes actores que desarrollan estrategias de intervención en prevención o castigo:

a) Estado: falta de acción de los estados que en muchas ocasiones se beneficia de la mano de obra barata para la agricultura, por ejemplo, o de las remesas provenientes del extranjero. Igualmente inactividad en las instituciones públicas que controlan el trabajo, la migración, o que desarrollan procesos educativos o de salud, entre otros.

b) Ministerio Público: la impunidad, la ausencia de políticas, de legislaciones o el no implementar leyes que castiguen la trata de personas. Falta de definición o claridad del delito. También la violación a los derechos humanos que no es otra cosa que incumplimiento de los estados.

c) La tolerancia social por desconocimiento de las situaciones de vulnerabilidad que van muy asociadas con condiciones de pobreza, de ignorancia, de necesidad de empleo, de sueños, de migraciones y de estereotipos sobre las víctimas.

En este marco posible de estadios de acción para la intervención en prevención se muestran actores como el estado y su institucionalidad (Ministerio Público, Ministerio de Educación), organizaciones no gubernamentales y la sociedad civil. Actores que se mueven como una red en la 
que una modificación en el trato del problema afecta directamente a los otros actores. Así, un cambio significativo en la educación sexual o en el desarrollo de destrezas emocionales repercutirá en las personas jóvenes, quienes podrían también impactar a su núcleo familiar. Se esperaría entonces, un cambio en todos los grupos que educan (docentes y cabezas de familia) y en quienes son educados (estudiantes e hijos).

Para trabajar una prevención integral de derechos que involucre personas jóvenes, la familia, centro educativo y comunidad, se debe reconocer previamente las construcciones sociales o estereotipos que estigmatizan actos individuales o grupales como las trabajadoras sexuales. Muchos de esos estereotipos son condiciones necesarias para potenciar o al menos ignorar el problema de la trata y, consecuentemente, no denunciar y no prevenir a personas en condiciones de vulnerabilidad.

Los estereotipos fijan una realidad, legitiman $\mathrm{y}$ hacen pasar por verdaderos los mitos que abundan sobre la temática, verbigracia: "todas las víctimas de trata son mujeres, niños o niñas"; "no hay trata en mi país", "todas cruzan la frontera, solo le sucede a las personas extranjeras en condición irregular", "en la trata solo hay explotación sexual", "solo les sucede a las personas pobres y sin estudio", "siempre son raptadas", "las prostitutas hacen lo que hacen porque quieren y pueden salir de su situación si quisieran, además les gusta lo que hacen".

Este sentido común, lamentablemente impregna a la sociedad, al personal de las instituciones públicas, docentes, policías, fiscales, jueces entre otros, impidiendo que se realicen acciones tendientes a la prevención, denuncia o castigo de las mismas. Se convierten en actores-actoras pasivas de una realidad que involucra a cualquier persona sin un momento determinado.

Es por ello que se considera que una estrategia de prevención debe promover información que permita identificar objetivamente el problema, las situaciones de vulnerabilidad que por una razón etaria, social, política, económica o cultural les pone en situaciones de riesgo, así como el hecho de que la intervención debe promover destrezas que le permitan a la población identificar un problema de trata y buscar y dar al menos opciones de solución.

Por lo tanto, la estrategia integral de prevención que se sugiere parte de un enfoque de derechos humanos fundamentales, por la intencionalidad de cubrir el derecho a la vida, a la autodeterminación, a tener nombre y nacionalidad; el honor, la dignidad, la libertad y la salud entre otros. La integralidad, además de conocer los derechos individuales, implica el desarrollo de destrezas, habilidades y capacidades frente al problema de la trata.

Desde el aprendizaje basado en competencias (Tobón, 2006), gracias al pensamiento complejo que le es inherente, el ser humano puede construir o desarrollar su capacidad de sentir, reaccionar, hacer, comprender, resistir, redefinir sus pensamiento y plantear nuevas estrategias frente a lo real. El pensamiento complejo "constituye un método de construcción del saber humano desde un punto de vista hermenéutico, o sea, interpretativo y comprensivo, retomando la explicación, cuantificación y la objetivación" (Tobón, 2006: $3)$. Este proceso permite la interacción personal desde diferentes dimensiones de la complejidad de lo real a partir de un pensamiento reflexivo. Es hermenéutico porque los sentidos ayudan a la experimentación y el consecuente conocimiento y la formación del sentido de la vida se activan en procesos deductivos, inductivos, análogos o de abducción propios del ser humano y que, en nuestro caso particular, en el que buscamos resolver un problema de trata de personas, el desarrollo de competencias centradas en la persona "y en su capacidad de aprender, que exige más protagonismo y un compromiso efectivo, debido a que la propia persona, debe desarrollar la capacidad de manejar la información original, buscando compararla, seleccionarla y evaluarla utilizando diversas modalidades" (Beitone y otros, 2007: 43).

El pensamiento complejo se relaciona con el diseño curricular basado en competencias, en tanto que ambos son el resultado de una investigación que permite identificar las competencias 
que se requiere formar para que la persona pueda resolver el problema de intervención.

Un proceso en formación centrado en la persona, como lo es la formación basada en competencias, permite el desarrollo de las capacidades internas para el control de sí misma, de su entorno y, consecuentemente, para modificar conductas y tomar decisiones. Las competencias permiten la interacción permanente entre lo individual y lo colectivo. Se parte de la realidad problematizada que está en continuo cambio y se transita por la organización de todo el aprendizaje, integrando los aspectos cognitivos, actitudinales y procedimentales.

Es por ello que la propuesta parte del trabajo con población joven, para que en lo cognitivo, logre caracterizar las condiciones de vulnerabilidad que le afecta directamente por su condición etaria (falsa idea del triunfo, falsa idea de la felicidad, sus afectaciones emocionales propias de la edad, manejo del poder entre pares, derechos especiales, entre otros). Las destrezas por desarrollar en este proceso de formación deben apuntar básicamente a identificar en lo cotidiano las situaciones de riesgo que puedan desembocar en un reclutamiento por engaño o por uso de la fuerza o coerción y junto a esta destreza, también saber qué hacer y cómo resolver esa situación. Dentro de los valores que deben desarrollar están la autodeterminación, la libertad, la dignidad humana y la felicidad.

Como componente externo de la estrategia de formación están las instituciones y personas de la comunidad, las que deben a su vez desarrollar esas mismas destrezas y habilidades para ayudar a la población joven a que no caiga en situaciones de riesgo. Deberán saber y reconocer que las personas jóvenes son sujetos integrales con condiciones particulares que deben ser atendidas como, por ejemplo, educación, alimentación, empleo, y no menos importante que todas estas redes contextuales de las personas jóvenes vitales para su desarrollo, deben mirar a estas y estos últimos con nuevos lentes, que les permita superar las viejas ideas de la juventud como problemas, rebeldes o sin derechos, y verles y reconocerles como seres humanos exactamente con los mismos derechos.

La habilidad que debe desarrollarse en la comunidad en general, es la de denunciar y erradicar las situaciones de demanda de todo tipo de esclavitud laboral, sexual, adopciones ilegales, tráfico de órganos, etc.

Es por ello que la estrategia integral de prevención interviene en dos momentos, con población (incluida la joven) y la comunidad. En la primera se trabaja por un proceso integral modular de formación basada en competencias para la prevención de situaciones de riesgo y de identificación y solución de problemas; y en el segundo momento, en un proceso de información a la comunidad que promueva la erradicación de todo tipo de demanda y situación de vulnerabilidad.

\section{Propuesta metodológica}

El trabajo desde la prevención con un enfoque de derechos implica desarrollo de habilidades y competencias y conocimiento y ejercicio de derechos, como vimos anteriormente. Por ello, el planteamiento metodológico parte de un proceso de aprendizaje e interaprendizaje, de intercambio de ideas y de opiniones, tal como afirma Gutiérrez:

es trans-formación, es com-unión, por lo que se da y por lo que se recibe. Es inter-acción como también lo es la co-municación... El aprendizaje que siempre es cambio, es la contrapuerta que nos demuestra si nos estamos o no comunicando, es decir, si en la comunicación nos estamos educando unos/ as y otros/as. Esto supone que aprende tanto ellla comunicadorla, al identificar la realidad, como ellla perceptorla al descodificar esa misma realidad comunicada. Educación y comunicación es un mismo y único proceso de co-participación, de co-producción, de co-entendimiento y co-munión (1989: 5).

La comunicación es indispensable en el proceso por seguir, es la base para la transformación de ideas en acciones en las que todas las personas involucradas están en una misma sintonía y manejando una terminología común, máxime cuando se trata de prevenir situaciones de violencia. 
Según plantea Abarca citado por Ramellini (2004:13-18), el interaprendizaje bajo una metodología participativa, incorpora tres momentos: a) partir de la práctica, b) la teorización y c) la vuelta a la práctica.

El primer momento se refiere a la importancia de partir de las propias personas, de sus conocimientos sobre la realidad, sus dificultades y problemas, de lo que les es común y hasta cotidiano, de manera tal que durante el proceso puedan dar significado y sentido a la experiencia y conocimientos adquiridos.

Según Ramellini (2004:14), este primer momento pedagógico es imprescindible para:

- Romper con la tradicional educación desde la proclama y el discurso, para construir una forma alternativa desde las demandas, necesidades, sufrimientos y vacíos de las personas.

- Romper el esquema "de la o el que sabe" y enseña "a la o el que no sabe", depositando conocimientos.

- Realizar una adecuación al tipo de participantes y la realidad en que actúan, de modo de dar utilidad, sentido y significación al proceso educativo para los/as mismos/as.

Para dicha autora (2004:14), el segundo momento, la producción colectiva de conocimientos, se fundamenta en que el saber no es propiedad de los/as intelectuales y académicos/as, ni algo finalizado y permanente que puede ser transmitido por una persona a la conciencia de otra. Nos hace ver que el interaprendizaje debe considerarse como algo inacabado, vivo, dinámico que se desarrolla por medio de la conversación y la reflexión conjunta. Los conocimientos se generan a través del proceso educativo de forma conjunta entre todas las personas participantes coordinados por un/a facilitador/a, y teniendo un referente teórico acuñado por la experiencia en el campo.

Para la construcción colectiva de conocimiento se necesitan de ciertas condiciones que faciliten y promuevan este proceso, entre las que se citan:
- Ambiente y actitudes humanas adecuadas: implica desarrollar un clima de confianza entre las personas participantes.

- Pensamiento crítico: para ello hay que promover el diálogo crítico en los temas por debatir y analizar.

- La participación consciente y activa: por lo que hay que promover entre las personas participantes el aporte de ideas enriquecedoras en el debate e incentivar la participación aunque las personas crean que esas ideas no son relevantes.

- La creatividad: se construyen conceptos propios de acuerdo con las experiencias vividas.

- Aprovechar los conocimientos y experiencias acumuladas: implica utilizar el conocimiento existente para cuestionarle y darle un nuevo sentido de acuerdo a la realidad circundante.

- La apropiación de conocimientos: las personas participantes deben de apropiarse del conocimiento, de manera tal que lo integren en su accionar y les permita reproducirlo.

- Una facilitación efectiva: la persona que coordina esta actividad debe favorecer la creatividad, promover el orden de ideas, hacer preguntas que conduzcan a la reflexión y el cuestionamiento sin perder ni dejar de lado el objetivo que se persigue (Ramellini, 2004:15-16).

El tercer momento metodológico, la vuelta a la práctica en la metodología participativa, promueve la transformación de la realidad de las personas participantes, de manera tal que se mejore, cambie y transforme para llenar vacíos y satisfacer necesidades.

Para Abarca, hay varias formas de realizar la vuelta a la práctica en el interaprendizaje, entre ellas:

- La elaboración de propuestas alternativas acordes a las posibilidades del grupo.

- El diseño y ejecución de planes y programas de acción.

- Realización de movilizaciones, foros, mesas redondas, debates, apertura de espacios para encontrar soluciones a los problemas vividos. 
- Solicitar la visita de otras personas conocedoras de las problemáticas vividas, para conjuntamente encontrarle salida a las necesidades (Ramellini, 2004:16-17).

Teniéndose como sustento teórico lo anterior, los procesos de interaprendizaje para la prevención de situaciones de violencia deben, necesariamente

partir del principio básico de que los procesos educativos son procesos de comunicación. Si estamos de acuerdo con este principio, convendremos en que mejorar la calidad de la comunicación inherente a los procesos educativos, es mejorar la calidad de los procesos de interaprendizaje ... la calidad del interaprendizaje viene dada por la calidad de las relaciones comunicacionales entre los actores/as del proceso (Ramellini, 2004: 20-21).

Apelando a la relevancia de favorecer las relaciones de comunicación entre las partes es que se promueve la facilitación del intercambio de ideas entre actores participantes según grupos poblacionales, de manera tal que cada cual se sienta a gusto para desarrollar la estrategia metodológica sugerida.

\section{Abordaje, desde la UNED, de la temática de la trata de personas}

La UNED ha venido realizando esfuerzos para incorporar el tema de la prevención y atención de la trata de personas desde diversos ámbitos como el académico, la extensión, la creación de la Comisión Institucional para la Prevención de la Explotación Sexual Comercial en Niños, Niñas y Adolescentes (CI-PESCNNA) y en la integración por parte de la UNED en el Capítulo Nacional del Observatorio Sobre Trata de Personas.

En docencia se rescata el esfuerzo de diferentes instancias académicas, como el caso del Proyecto Intercátedras Es Asunto de Todos y de Todas perteneciente a la Escuela de Ciencias Sociales y Humanidades. En dicho Proyecto, diferentes cátedras han incluido el tema de la trata de personas en las acciones formativas dirigidas a estudiantes, mediante tareas, proyectos, foros, talleres, entre otros, los cuales han permitido que ellos mismos investiguen sobre la temática y realicen acciones preventivas en sus comunidades.

Por su parte, la Comisión Institucional para la Prevención de la Explotación Sexual Comercial en Niños, Niñas y Adolescentes (CIPESCNNA) creada por el Consejo de Rectoría, en sesión $n .^{\circ}$ 1679-2011, artículo III, inciso 11, celebrada el 30 de mayo de 2011, expone su objetivo general:

Promover las acciones institucionales pertinentes en la temática de la prevención de la explotación sexual comercial en niños, niñas y adolescentes, así como también abordar el tema de la trata y trabajo infantil; para sensibilizar a la comunidad universitaria y desarrollar actividades comunales que promuevan las actitudes protectoras en la sociedad costarricense" (CI-PESCNNA, 2011: 3).

Dicha Comisión ha realizado esfuerzos preventivos no solo en la sede central, sino también en los diferentes centros universitarios mediante la organización y participación en talleres, charlas, foros, capacitaciones, videoconferencias y otras acciones tendientes a sensibilizar a la población en el tema de explotación sexual comercial y la trata de personas.

Asimismo, la UNED, junto con otras universidades estales como la Universidad de Costa Rica, la Universidad Nacional y organismos no gubernamentales como la OIM, CEFEMINA y MUSADE inician en febrero del 2013 conversaciones con miras a la conformación del Capítulo Nacional del Observatorio Sobre Trata de Personas (observaLaTrata), que se adscribe al Observatorio Latinoamericano de Trata de Personas, cuyo objetivo es contribuir a la investigación acerca de la trata de personas a nivel latinoamericano bajo un enfoque de género y de derechos humanos. Entre otros, sus ejes de observación son la jurisprudencia, el acceso a la justicia y los medios para el delito mediante la construcción de indicadores de observación generales y particulares de cada país. 
El Capítulo Nacional se conforma durante el I Foro Nacional de Trata de Personas (4 junio de 2013), en el cual la UNED asume la subcoordinación del observaLaTrata -la coordinación la ocupa la UCR- y se compromete a realizar acciones tendientes a prevenir e informar a través de la investigación, la docencia y la extensión con miras a contribuir a la erradicación de la problemática social.

Entre las acciones ejecutadas por el Capítulo Nacional están el Conversatorio sobre buenas prácticas y lecciones aprendidas para la aplicación de la Ley contra la Trata de Personas, charlas acerca de la realidad de la trata de personas en México y el mundo, la visita del presidente del observaLaTrata Latinoamérica, Óscar Castro, el I Foro Nacional de Trata de Personas y el foro Retos y desafíos a un año de la aprobación de la ley contra la trata de personas.

A lo anterior, se suman las gestiones y tareas ejecutadas por parte de cada una de las instituciones integrantes del Capítulo Nacional, que contribuyen al cumplimiento de los objetivos propuestos en el plan de trabajo del observaLaTrata.

\section{Aportes al tema desde Agenda Joven}

En la UNED se han desarrollado diferentes procesos de formación respecto del tema de la trata de personas específicamente desde el programa Agenda Joven. En esta línea, esta instancia en alianza estratégica con la Organización Internacional para las Migraciones (OIM) diseñó en su plataforma un curso virtual con una duración de diez semanas. La propuesta pedagógica integra un proceso de interaprendizaje, el constructivismo y la educación a distancia.

El interaprendizaje parte de

una concepción pedagógica que promueve la participación y la vivencia permanente como formas para lograr la interiorización del significado de sus vidas y la apropiación temática... Valora la experiencia y conocimientos adquiridos por las personas en sus vidas, trabajos y relaciones establecidas... Reconoce y valora la dimensión subjetiva, emocional y afectiva de las personas como la base para construir el respeto, la tolerancia y la equidad entre ellas (OIM, s.f.: 8).

De este modo, es posible trabajar actitudes personales o sociales respecto del tema que responden a mitos, prejuicios, y se espera que los estudiantes puedan hacer una revisión de estos y posteriormente transformarlos con miras a la prevención y protección real de las víctimas. Consecuentemente, hay un componente ético de formación para la transformación del entorno y el prisma para mirarlo. Con el constructivismo se da una elaboración grupal de los conceptos y temas, en donde cada estudiante parte de su experiencia, con actitud crítica y propositiva.

El curso desarrolla aspectos básicos de la trata de personas, las condiciones previas, la etapa de captación, el traslado, la explotación, la salida y la reintegración. También el impacto de la trata en las sobrevivientes, la atención a víctimas y la prevención integral.

La investigación Percepciones docentes sobre la trata de personas (2014), desarrollada en Agenda Joven indaga los siguientes aspectos sobre el manejo de estereotipos de la trata: conocimiento docente de la temática y los fines de la trata de personas por medio de escala Likert, y en cuanto a la estrategia de prevención, las competencias y destrezas a desarrollar.

Es importante destacar los aspectos más importantes de esta investigación:

1. Hay una percepción del $63 \%$ en los y las docentes que indican que pueden ser víctimas de trata, dato que se condice con el $56 \%$ que manifiesta que en su comunidad existe este problema. La trata de personas no es un problema ajeno a cada uno de ellos. Lo viven como una posibilidad cotidiana. Este temor podría extrapolarse a otros miembros de la familia y la comunidad.

2. Hay un conocimiento claro de lo que es la trata de personas y de los diferentes tipos que existen. Sin embargo, cuando se hace referencia a la trata por mendicidad y la trata con fines militares se presentan dudas sobre si la misma existe o es posible. 
3. La pobreza, el bajo nivel académico y la migración son condiciones que se señalan como las de mayor vulnerabilidad y, por consiguiente, se identifica a esta población como la que corre mayor riesgo. Contraponer este dato con su propia percepción de ser posible víctima podría señalar que los estereotipos que se ciernen sobre las víctimas son un punto o tema que debe trabajarse en una estrategia de intervención para la prevención.

4. Los lugares que se señalan como aquellos en los que se puede dar la trata de personas no se puntualizaron, sino que los docentes prefirieron caracterizarlos. Se manifestó que es posible la trata en zonas con problemas sociales y económicos y en zonas internacionales y fronterizas. En este sentido se visualiza correctamente el proceso de captura, traslado y victimización de las personas tratadas.

5. Hay una percepción correcta sobre los derechos más vulnerados como el derecho a la libertad y a la dignidad, y se reconoce la responsabilidad que tiene el Estado para generar estrategias de prevención, especialmente el MEP, el PANI, la Fuerza Pública y el OIJ.

6. La trata de personas se da por comercio y lucro $(40,3 \%)$ y por pérdida de valores morales y religiosos $(33 \%)$. Hay un porcentaje elevado de $33 \%$ que saca el problema de la trata de personas de su contexto material-comercial, con lo que resta la responsabilidad del victimario al insinuar que sus actuaciones responden a mandatos sociales "alterados", es decir, a valores modificados respecto a los de antes. Supone a la vez este razonamiento que antes de que se diera el cambio o "pérdida" de valores la trata de personas no existía. Sabemos que la trata de personas es un problema antiguo y que la demanda es el problema.

\section{Propuesta desde la UNED}

Bajo el panorama anteriormente expuesto, Costa Rica, al igual que otros países, está expuesta a la trata de personas en sus variadas manifestaciones, por lo que se debe tener conocimiento actual de la situación por la que atraviesa el país en ese sentido, con el fin de implementar políticas públicas que contribuyan a erradicar el problema. De ahí que sea imprescindible la realización de un diagnóstico situacional que afirme las bases para futuras estrategias de acción de incidencia preventiva, de atención o de coordinaciones interinstitucionales, componentes todos del Plan Estratégico de Trabajo de la Coalición Nacional contra el Tráfico Ilícito de Migrantes y la Trata de Personas: Costa Rica 2012-2015.

Costa Rica se ubica geográficamente en el centro de América, de forma tal que se puede convertir tanto en puente como país de paso para la migración con fines de trata, o bien, como nación para la explotación y trata de personas, de ahí el que se requiera conocer la realidad para generar las acciones pertinentes. No se puede obviar que Costa Rica ha sido señalado como "el país más conocido y 'afamado' como paraíso sexual entre los países de la región" (UNODC, 2009:20) y por ende, una nación en la que el tráfico de personas se puede acentuar y proliferar.

Según el "Informe regional del alcance del problema y la respuesta gubernamental y no gubernamental" preparado por The Protection Project de la Universidad Johns Hopkins (School of Advanced International Studies) referente a la trata de personas, en especial mujeres y niños en los países de América $^{3}$, se indican esfuerzos realizados en los diferentes países que incluyen

3. El informe fue realizado en los siguientes países: Antigua y Barbuda, Argentina, Bahamas, Barbados, Belice, Bolivia, Brasil, Canadá, Chile, Colombia, Costa Rica, Cuba, Dominica, República Dominicana, Ecuador, El Salvador, Estados Unidos, Grenada, Guatemala, Guyana, Haití, Honduras, Jamaica, México, Nicaragua, Panamá, Paraguay, Perú, St. Kitts and Nevis, St. Lucia, St. Vincent and the Grenadines, Suriname, Trinidad y Tobago, Uruguay y Venezuela. 
campañas masivas en los medios de comunicación para crear una conciencia pública sobre problema de la trata, conferencias regionales que abordan varios aspectos de la trata, grupos de trabajo gubernamentales especializados en combatir el trabajo infantil y otros problemas relacionados con la explotación de personas menores de edad, Planes $\mathrm{Na}$ cionales de Acción para combatir el problema de los niños y niñas de la calle, varios programas "permanezca en la escuela" y otros tipos de reformas educativas y algunas iniciativas de los gobiernos para combatir la explotación sexual, revisión de los procesos de adopción y abordaje de la problemática del trabajo infantil (s.f.).

Se señala que los esfuerzos deben continuar de forma sistemática y en apoyo conjunto entre las diferentes naciones, se propone

elementos de una legislación modelo que los países del hemisferio puedan considerar para modificar las leyes existentes o promulgar nuevas leyes contra la trata... debido a que la trata de personas es una práctica común entre los países del hemisferio, todo esfuerzo que se haga para combatirla debe comprender políticas regionales y trasnacionales dedicadas a establecer cooperación entre los paises para arrestar, encarcelar y enjuiciar a los responsables por el acto de la trata (s.f.).

Según datos del "Diagnóstico de las capacidades nacionales y regionales para la persecución penal del delito de trata de personas en América Central" (Comisión Nacional de los Derechos Humanos y otro, 2009), Costa Rica es el país que presenta menor cantidad de denuncias (cinco en total) entre los años 2003 y 2008; y de estos casos solo uno tiene sentencia condenatoria. El país con mayor número de denuncias en el mismo periodo es El Salvador (178), quien a su vez presenta para el 2008 mayores denuncias condenatorias (11). La ausencia de menor número de denuncias en nuestro país podría ser, entre otras razones, reflejo del subregistro de la información por la presencia de amenazas a familiares y víctimas o por una errónea tipificación del delito, de ahí el que aumente la necesidad de realizar un diagnóstico nacional que actualice datos y determine la realidad.
En otros países latinoamericanos como México, Paraguay, Ecuador y Panamá ya se han realizado diagnósticos que han apoyado propuestas dirigidas a la prevención e intervención de este flagelo.

En el caso de México hay varios diagnósticos relacionados con la problemática, como el "Diagnóstico sobre la trata de mujeres y niñas en el Estado de México" (s.f.), el "Diagnóstico de las condiciones de vulnerabilidad que propician la trata de personas" (2009), el "Diagnóstico de causas estructurales y sociales de la trata de personas en la ciudad de México: Propuesta de Programa para prevenir, sancionar y erradicar los delitos en materia de trata de personas y para proteger y asistir a las víctimas en el Distrito Federal 20132019” (2012) y "La Trata de Personas en México: Diagnóstico sobre la asistencia a víctimas" (2011).

Respecto a este último documento se destacan algunos datos relevantes como el hecho de que

el fenómeno de la trata responde a diversos factores relacionados, principalmente, con la oferta $y$ demanda de mano de obra barata, las desigualdades económicas y problemas socioculturales aún no resueltos, como la discriminación y la violencia de género. Otras cuestiones, como la ubicación geográfica o el contexto migratorio general, influyen también en la incidencia del delito (OIM, 2011:9).

Por otra parte, la Organización Internacional para las Migraciones (OIM), ente financiante del documento anteriormente citado, y dado su compromiso con la problemática, financia otras investigaciones similares como la de Paraguay denominada "La trata de personas en el Paraguay: Diagnóstico exploratorio sobre el tráfico y/o trata de personas con fines de explotación sexual", cuyos resultados permitieron

conocer cómo funciona en el país la captación y reclutamiento de las víctimas, los mecanismos utilizados, las rutas internas y externas. El diagnóstico señala los lugares donde diariamente se producen los más graves atropellos a la dignidad humana en las personas de niñas, adolescentes, jóvenes y adultas, que no dejan margen para desentenderse del problema (OIM, 2005). 
En Ecuador, la OIM y la FLACSO realizaron en el 2012 un diagnóstico de la situación de trata de personas en el cantón de Ibarra, provincia de Imbabura, al estar situado al norte de país y recibir constante migración de personas, mayoritariamente de origen colombiano. Ambos entes presentaron la necesidad de realizar una investigación de la temática por las condiciones y características propias de esa región del país. Por ello,

el diagnóstico hace una caracterización de la trata de personas en el cantón de Ibarra y las respuestas de actores públicos y privados frente a este fenómeno... determinar cuáles son las modalidades de trata de personas que afectan al cantón y cuáles son las acciones que realizan esos actores en términos de prevención, protección de las víctimas e investigación y sanción. A partir de esta información se realizan algunas recomendaciones hacia la construcción de una política contra la trata de personas a nivel local (OIM-FLACSO, 2012: 11).

Recientemente, en Panamá la Comisión Nacional Contra la Trata de Personas presentó los resultados del "Estudio sobre la situación de trata de personas en Panamá" (2014), que analiza las dimensiones, características e impacto de este delito y la respuesta de las instituciones en materia de protección y asistencia:

Los resultados revelaron que las modalidades de explotación más frecuentes son la laboral y la sexual, aunque también se identificó el reclutamiento para cometer delitos menores o de bajo nivel, la servidumbre en el matrimonio y la falsa adopción de niños. Entre los principales hallazgos, se observó la presencia de mujeres colombianas jóvenes para la trata sexual en clubes nocturnos; de mujeres nicaragüenses y dominicanas explotadas en servidumbre doméstica; población de nacionalidad china obligada a trabajar durante extensas jornadas en condiciones pésimas en mini-súpers y lavanderías, así como hombres colombianos y libaneses obligados a laborar sin respeto a sus derechos humanos en restaurantes y otros servicios de hostelería.

Los diagnósticos anteriormente citados y otros esfuerzos que al respecto se han realizado en los diferentes países han servido de base para la direccionalidad de las propuestas y la priorización de las mismas, así como para otros proyectos e investigaciones que surjan en segundos momentos.

Partiendo del panorama anteriormente descrito, la realización de un diagnóstico sobre la situación de trata del país deberá ser participativo, con enfoques basados en los derechos humanos y los estudios de género, para promover la integralidad y la inclusividad, de forma tal que se logren articular las diferentes visiones y cosmovisiones que al respecto de la trata de personas se tengan.

La UNED brinda la posibilidad de realizar el diagnóstico a nivel nacional utilizando como plataforma los 37 centros universitarios y subsedes que tiene distribuidos según la división territorial administrativa del país (ver Figura 1), lo cual brindará una caracterización general bastante amplia de las seis regiones, y permitirá reconstruir posteriormente los resultados a nivel nacional.

Es importante destacar que la cantidad de centros universitarios y subsedes que tiene la Universidad facilita el abarcar un amplio porcentaje de la población en la que estos tienen incidencia, promoviéndose a su vez la divulgación e invitación para la asistencia de las personas a las actividades convocadas, con miras a obtener un mayor impacto.

Bajo este panorama de plataforma universitaria, se pretende trabajar en talleres que convoquen a niñez, adolescencia, profesionales en diversos campos y sociedad civil interesada en la temática, con el fin primordial de realizar, además del diagnóstico de trata, situaciones acontecidas, estrategias de atención e intervención de las instituciones asistentes, recursos, fortalezas y debilidades por sector consultado, entre otros asociados a esta temática.

En cada taller, se trabajará por separado y con técnicas participativas según población convocada las mismas temáticas. Para ello se crean cinco momentos:

1. Conocimientos previos asociados a la trata de personas.

2. Presentación teórica y desmitificación de la temática, así como el abordaje de la prevención bajo un enfoque de derechos. 


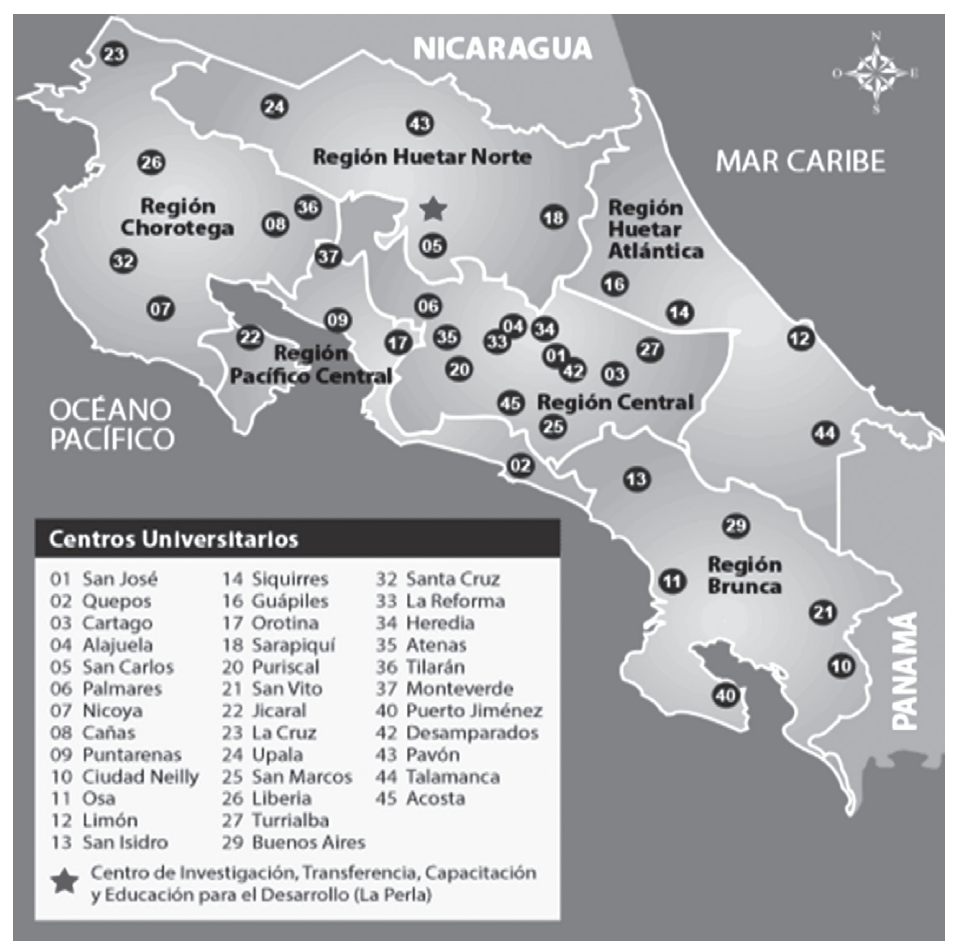

Figura 1. Centros universitarios y subsedes de la UNED. Fuente: Página web de la UNED, 2014.

3. Trabajo colaborativo, para que, desde su propia realidad (etaria, social o profesional) realicen una propuesta de prevención en la materia, que sea integral, atractiva y que pudiera tener impacto en la población.

4. Socialización de la propuesta de prevención por grupo convocado, de manera tal que se analicen aciertos, desaciertos, concordancias, similitudes y desavenencias, y se pueda crear una propuesta de prevención abarcativa y de impacto para la población en general.

5. El último momento incluye el compromiso de trabajar a partir de la temática, para convertirse en líderes y multiplicadoras de la experiencia de prevención que se establezca para ese sector del país.

Como producto final se elabora una propuesta general de prevención por cada una de las zonas representadas en los centros universitarios que tiene la UNED.

Con la información recopilada en estos talleres, desde el grupo de trabajo central, se realiza una propuesta de prevención nacional integral para ser desarrollada por las comisiones establecidas anteriormente.

Esta propuesta final de prevención se puede implementar a través de los servicios y recursos con que cuenta la UNED, a saber: videoconferencias, trabajo en plataforma, programas radiales, cortos televisivos, entre otros, lo que indudablemente representa coordinar previamente con estas instancias.

Se considera que esta estrategia metodológica de prevención se puede realizar simultáneamente en varios centros universitarios, lo que favorece el intercambio de ideas y conocimientos, acrecienta el interaprendizaje y favorece la reflexión acerca de este tipo de violencia, en aras de fortalecer la 
prevención y encauzar la atención hacia quienes requieren de la misma.

Finalmente, y como consecuencia de lo anterior, se requiere una UNED comprometida con la prevención de la trata de personas, que asuma la temática como un reto, en donde las personas funcionarias se involucren en la totalidad o en parte con lo propuesto, que se destine tanto recurso humano como técnico, tecnológico y financiero para cumplir con las etapas de la estrategia antes descrita, y esto, definitivamente, está en la voluntad y el compromiso que asumamos. Esto es lo que, al final de los procesos de esta índole, le brinda la sostenibilidad y seguimiento a la investigación y a los resultados que a través de esta se obtengan.

\section{A modo de conclusiones}

La trata de personas es una forma moderna de esclavitud y su problema se origina en la demanda. Hay muchas situaciones que generan condiciones de vulnerabilización de posibles víctimas, las cuales pueden ser materiales y objetivas, como el engaño, el uso de la fuerza, la amenaza o la coacción. La demanda de cualquiera de las formas de esclavitud es el origen de la cadena de victimización y deshumanización de la persona tratada. La persona se cosifica al perder su condición de ser humano integral y sujeto de derechos. El sometimiento de las personas es posible por el uso de las amenazas o por el desarraigo de la víctima, que incluye el despojo de sus papeles migratorios en los casos de tráfico internacional.

Toda estrategia de prevención debe plantearse el papel que jugarán el Estado y la ciudadanía en general, porque la trata de personas es tanto delito penal como imaginario estereotipado de la ciudadanía hacia las personas; en el primer caso, el problema radica en que no se reconoce el delito en las legislaciones internas y no hay sanciones por el delito propiamente dicho, sino por secuestro o explotaciones; y en el caso de la ciudadanía, lo que intervienen son las ideas que se han fijado en estereotipos que impiden a las personas identificar las situaciones de riesgo y el delito mismo. Una sociedad con estas características permea a la institucionalidad en todas sus formas y representaciones. En este orden, la estrategia de prevención debe integrar el enfoque de derechos, el enfoque de género y el interaprendizaje.

El enfoque de derechos y persona integral ubica la trata en la dimensión correcta: un delito cometido contra un ser humano. La interrelación derecho e integralidad apunta a la materialización en la protección de derechos de las personas para resguardar su integridad física, psicológica y social y, sobre todo, para resguardar su capacidad para autodeterminarse y tener una vida plena.

El interaprendizaje como referente metodológico permite la apropiación e interiorización de los principales componentes de la trata en tanto la persona le da significado según su propia experiencia, y ello implica una revisión personal de los estereotipos que se asocian a la trata personas.

Los diagnósticos realizados en los diferentes países referentes a la trata de personas han anotado la importancia y necesidad de ejecutar los mismos, ya que sirven de base para la formulación o fortalecimiento de políticas públicas, o bien, para el desarrollo de planes, programas o proyectos específicos que contribuyan a combatir la problemática en cuestión. Para el caso Costa Rica, se hace imprescindible la elaboración de un diagnóstico de trata de personas, para no solo elaborar propuestas concretas, sino también para direccionar los recursos según las necesidades específicas que se tengan a nivel cantonal-regional-nacional.

La utilización de la plataforma de la UNED mediante los diferentes centros universitarios, sedes y subsedes, facilita el proceso investigativo, ya que se parte de un ente familiar para las comunidades y regiones, que permitirá el acercamiento necesario para alcanzar el objetivo propuesto.

Contar con el apoyo de la Coalición Nacional Contra el Tráfico Ilícito de Migrantes y la Trata de Personas es un factor determinante para el éxito de cualquier iniciativa de esta índole que se vaya a realizar, ya que se asegura el respaldo financiero y técnico necesario para impactar en las políticas públicas que a futuro se desarrollen, así como en la formulación de planes y proyectos específicos para abordar la 
temática según las necesidades reales sentidas en cada región del país.

La UNED, más allá de ser una institución abanderada en la defensa de los derechos humanos, es un ente comprometido con la población vulnerable y susceptible de ser víctima, en aras de contribuir, desde diversas áreas, a combatir y erradicar la trata de personas en Costa Rica.

\section{Bibliografía}

Agenda Joven (2014). Percepciones docentes sobre la trata de personas. San José: UNED.

Batista, A. (s.f.). "La trata de personas, esclavitud del siglo XXI". Recuperado de < http://www.feminamericas.net/ ES/tematicas/PRES-AllietBautista-traite-e.pdf>.

BID (2006). "Las sucias y costosas ganancias de la explotación humana". Recuperado de <http://www.iadb.org/ es/noticias/articulos/2006-11-02/las-sucias-y-costosasganancias-de-la-explotacion-humana,3357.html>.

Beitone, P., C. Esquetini, J. González, M. Marty, G. Siufi y R. Wagenaar (2007). Reflexiones y perspectivas de la educación superior en América Latina. España: Universidad de Deusto.

Ci-Pescnna (2011). Comisión Institucional para la Prevención de la Explotación Sexual Comercial en Niños, Niñas y Adolescentes: Informe Ejecutivo 2011-2012. San José: UNED.

Comisión Nacional De los Derechos Humanos \& Centro de Estudios e InVestigación en Desarrollo y AsistenCIA Social, A.C. (2009). "Diagnóstico de las condiciones de vulnerabilidad que propician la trata de personas". México. Recuperado de <http://imumi.org/attachments/ article/141/Diagnostico_Trata_de_Personas_CEIDASCNDH.pdf>.

Comisión Nacional Contra La Trata De Personas (2014). "Estudio sobre la situación de trata de personas en Panamá". Panamá: Gobierno Nacional. Recuperado de <http://www.ana.gob.pa/index. php/8-noticias/418-presentan-diagn $\%$ C3\%B3sticonacional-sobre-la-situaci $\% \mathrm{C} 3 \% \mathrm{~B} 3 n$-de-la-trata-de-personas-en-panam $\% \mathrm{C} 3 \% \mathrm{~A} 1 . \mathrm{html}$.

Dirección General De Migración Y Extranjería, Coalición Nacional Contra el Tráfico Ilícito de Migrantes y la Trata de Personas \& UNICEF (2012). Plan Estratégico de Trabajo de la Coalición Nacional contra el tráfico ilícito de migrantes y la trata de personas: Costa Rica 2012-2015. San José.
Gobierno Federal de México, Instituto Nacional de las Mujeres, Vivir Mejor, Gobierno del Estado de México, Consejo Estatal de la Mujer y Bienestar Social \& Proyectos Mujer A.C. (s.f.). "Diagnóstico sobre la trata de mujeres y niñas en el Estado de México". México. Recuperado de $<$ http://investigacion.politicas.unam.mx/catedratrata/wp-content/uploads/2013/10/edomexmeta4.pdf $>$.

Le Goff, H; T. Lothar \& OIM (2011). "La trata de personas en México: Diagnóstico sobre la asistencia a víctimas". Recuperado de <http://www.oim.org.mx/pdf/La\%20 Trata $\% 20 d e \% 20$ personas_diagnostico2.pdf $>$.

Gutiérrez, F. (1989). La comunicación como elemento fundamental en el mejoramiento de los procesos educativos. Heredia: Instituto de Pedagogía de la Comunicación (ILPEC).

Gobierno Nacional República De Panamá (2014). "Presentan Diagnóstico Nacional sobre la Situación de la Trata de Personas en Panamá". Recuperado de $<$ http://www.ana.gob.pa/index.php/8-noticias/418presentan-diagn $\%$ C $3 \%$ B 3 stico-nacional-sobre-la-situaci $\% \mathrm{C} 3 \% \mathrm{~B} 3 n$-de-la-trata-de-personas-enpanam $\% \mathrm{C} 3 \% \mathrm{~A} 1 . \mathrm{html}>$.

IPEC y Ort (2008). Sintesis Diagnóstico de situación del trabajo infantil y sus peores formas en Guatemala. Recuperado de <file:///Z:/Users/gvillalobost/Downloads/sintesis_ diagnostico_hoja_de_ruta_guatemala.pdf>.

Kant, I. (1996). Fundamentación de la metafísica de las costumbres. México: Porrúa.

Oıм (s.f.). Curso Virtual Sobre Trata de Personas. Manual para Tutores. San José.

Oim-Flacso (2012). "Diagnóstico de la situación de trata de personas en Ecuador en el cantón de Ibarra, provincia de Imbabura". Quito. Recuperado de <http://www.oim. org.ec/drupal/sites/default/files/Publicaciones/DIAGNOSTICO $\% 20$ TdP $\% 20$ OIM $\% 20$ IBARRA.pdf>.

OІм у Grupo Luna Nueva (2005). "La trata de personas en el Paraguay: Diagnóstico exploratorio sobre el tráfico y/o trata de personas con fines de explotación sexual. Paraguay”. Recuperado de <http://www.oas.org/atip/ Paraguay/Libro_Trata_Personas_Paraguay_spa.pdf $>$.

ONU (2003). Protocolo para prevenir, reprimir y sancionar la trata de personas, especialmente mujeres y niños, que complementa la Convención de las Naciones Unidas contra la delincuencia organizada transnacional (Protocolos de Palermo).

Ramellini, T. (2004). Módulo básico "para sentir, pensar y enfrentar la violencia de género, intrafamiliar y sexual". Taller de Interaprendizaje. San José: Inamu. 
Secretaria de Desarrollo Social de la Ciudad De MéXICO, Inmujeres DF y Coalition (2012). "Diagnóstico de causas estructurales y sociales de la trata de personas en la ciudad de México: Propuesta de Programa para prevenir, sancionar y erradicar los delitos en materia de trata de personas y para proteger y asistir a las víctimas en el Distrito Federal 2013-2019”. México. Recuperado de <http://www.equidad.scjn.gob.mx/IMG/pdf/DIAGNOSTICO_CAUSAS_SOCIALES_Y_ESTRUCTURALESTRAT.pdf>.

Tовón, S. (2004). Formación basada en competencias: pensamiento complejo, diseño curricular y didáctica. Bogotá: Ecoe Ediciones.
The Protection Project (School of Advanced International Studies), CEPAL (s.f.). Trata de personas, en especial mujeres y niños en los paises de América. Un informe regional del alcance del problema y la respuesta gubernamental y no gubernamental. Baltimore, Maryland: Universidad Johns Hopkins, Recuperado de <http://www.cepal.org/celade/ noticias/paginas/2/11302/MattarJohnHopkins.pdf $>$.

UnOdC e Ilanud (2009). "Diagnóstico de las capacidades nacionales y regionales para la persecución penal del delito de trata de personas en América Central". Recuperado de $<$ http://www.unodc.org/documents/human-trafficking/Diagnostico_regional_XCAS261.pdf>. 
